\title{
Use of $\beta$ blockers and the risk of death in hospitalised patients with acute exacerbations of COPD
}

\author{
M T Dransfield, ${ }^{1,2}$ S M Rowe, ${ }^{1}$ J E Johnson, ${ }^{1}$ W C Bailey, ${ }^{1}$ L B Gerald ${ }^{1}$
}

See editorial, p 296

${ }^{1}$ Division of Pulmonary, Allergy and Critical Care Medicine,

University of Alabama at Birmingham, Birmingham, Alabama, USA; ${ }^{2}$ Birmingham VA Medical Center, Birmingham, Alabama, USA

Correspondence to:

Dr M Dransfield, 215 THT, 1900 University Blvd, Birmingham,

Alabama 35294, USA;

mdransfield99@msn.com

Received 27 March 2007 Accepted 30 September 2007

Published Online First

19 October 2007

\begin{abstract}
Background: Cardiovascular disease is a major cause of death in patients with chronic obstructive pulmonary disease (COPD) and predicts hospitalisation for acute exacerbation, in-hospital death and post-discharge mortality. Although $\beta$ blockers improve cardiovascular outcomes, patients with COPD often do not receive them owing to concerns about possible adverse pulmonary effects. There are no published data about $\beta$ blocker use among inpatients with COPD exacerbations. A study was undertaken to identify factors associated with $\beta$ blocker use in this setting and to determine whether their use is associated with decreased in-hospital mortality.
\end{abstract}

Methods: Administrative data from the University of Alabama Hospital were reviewed and patients admitted between October 1999 and September 2006 with an acute exacerbation of COPD as a primary diagnosis or as a secondary diagnosis with a primary diagnosis of acute respiratory failure were identified. Demographic data, comorbidities and medication use were recorded and subjects receiving $\beta$ blockers were compared with those who did not. Multivariate regression analysis was performed to determine predictors of in-hospital death after controlling for known covariates and the propensity to receive $\beta$ blockers.

Results: 825 patients met the inclusion criteria. Inhospital mortality was $5.2 \%$. Those receiving $\beta$ blockers $(n=142)$ were older and more frequently had cardiovascular disease than those who did not. In multivariate analysis adjusting for potential confounders including the propensity score, $\beta$ blocker use was associated with reduced mortality ( $\mathrm{OR}=0.39 ; 95 \% \mathrm{Cl} 0.14$ to 0.99 ). Age, length of stay, number of prior exacerbations, the presence of respiratory failure, congestive heart failure, cerebrovascular disease or liver disease also predicted inhospital mortality $(p<0.05)$.

Conclusions: The use of $\beta$ blockers by inpatients with exacerbations of COPD is well tolerated and may be associated with reduced mortality. The potential protective effect of $\beta$ blockers in this population warrants further study.

Chronic obstructive pulmonary disease (COPD) is the fourth leading cause of death in the USA and the only major cause that is rising in frequency. ${ }^{1}$ Although many patients with COPD die from respiratory failure, cardiovascular disease is consistently the first or second leading cause of death depending on the severity of the participants' underlying lung disease and the individual study population..$^{2-6}$ Most of these cardiovascular deaths are due to coronary artery disease which is present in $10-22 \%$ of patients with COPD. ${ }^{78}$ This can be attributed to their high prevalence of traditional cardiovascular risk factors (eg, tobacco use, hypertension) and those more recently reported such as systemic inflammation and medication toxicity. ${ }^{7-12}$ Many COPD deaths occur during hospitalisation for acute exacerbations, a complication which carries an in-hospital mortality as high as $32 \%$ and predicts a 1-year mortality of $23-43 \%{ }^{13-18}$ Although these deaths are often attributed to underlying COPD, the contribution of cardiac disease is thought to be significant. ${ }^{15-17}$

Beta-blockers reduce mortality in patients with cardiovascular disease, most notably after myocardial infarction, ${ }^{19}$ in the presence of congestive heart failure ${ }^{20}$ and in the perioperative setting. ${ }^{21}$ Patients with COPD are often denied these medications because of possible adverse effects of $\beta$ blockade on airway function. ${ }^{19}{ }^{22}$ This practice continues despite the fact that studies with cardioselective $\beta$ blockers have shown the airway effect to be modest at most. ${ }^{23}$ A recent Cochrane review analysing 20 randomised trials of cardioselective $\beta$ blockers in COPD found no significant effect on forced expiratory volume in $1 \mathrm{~s}\left(\mathrm{FEV}_{1}\right)$ or bronchodilator response after single doses or up to 12 weeks of treatment. ${ }^{23}$ This was true even among patients with severe COPD and those with reversible airflow obstruction. Moreover, $\beta$ blockers have been shown to reduce mortality in patients with COPD with hypertension compared with other agents ${ }^{24}$ and to blunt the apparent cardiac toxicity of short-acting $\beta$ agonists. ${ }^{12}$ Although there has been more concern about the airway effects of non-cardioselective $\beta$ blockers, ${ }^{25}$ data are available that indicate that they are well tolerated in patients with COPD and congestive heart failure. ${ }^{26}$

Despite the apparent safety and potential benefit of $\beta$ blocker use in patients with COPD of all levels of disease severity, little is known about their effects during inpatient admissions for acute exacerbations where airway function is most severely compromised. The purpose of this study was to examine the use of $\beta$ blockers (both cardioselective and non-cardioselective) in patients admitted to a university hospital with acute exacerbations of COPD and to determine whether the administration of these drugs was associated with in-hospital mortality. We found that $\beta$ blocker use was an independent predictor of survival to hospital discharge.

\section{METHODS}

The computerised hospital record system was reviewed and patients admitted to our facility 
between 1 October 1999 and 30 September 2006 whose discharge or death summaries indicated a primary diagnosis of acute exacerbation of COPD (International Classification of Diseases, Ninth Edition (ICD-9) code 491.21) or a primary diagnosis of acute respiratory failure (518.81) and a secondary diagnosis of acute exacerbation were identified. Patients with a diagnosis of asthma (493) were excluded. Demographic data including age, race and sex; date of index admission (defined as the final admission during the study period); number of prior admissions for acute exacerbation of COPD during the study period; smoking status; attending physician of record and medical subspecialty service were recorded at the time of hospital admission. The presence of respiratory failure and comorbid illness including cardiovascular disease, diabetes mellitus, liver disease, chronic renal insufficiency and malignancy was determined by ICD-9 coding and the total disease burden was assessed using the Deyo-Charlson index. ${ }^{27}$ Lengths of stay and hospital disposition were also recorded. Inpatient medication use was determined by automated billing records. Patients were considered to have received a given agent if he or she was billed for any formulation of the drug at any time during the hospitalisation. For each drug the number of doses per hospital day was also calculated. Beta-blockers were categorised as cardioselective or non-cardioselective. We also reviewed records from our health system's pulmonary function laboratory and recorded a patient's spirometric data if they were present and obtained within 1 year of the index admission. The study was approved by the University of Alabama at Birmingham Institutional Review Board.

\section{Analysis of data}

Demographic data, diagnostic profile, medication use and inhospital mortality were compared between subjects who received $\beta$ blockers and those who did not. Continuous variables were compared using Student $t$ tests and categorical variables were compared using the $\chi^{2}$ or Fisher exact test as appropriate.

A propensity score technique was used as an additional method to balance covariates associated with $\beta$ blocker use between groups. ${ }^{28}$ The propensity score was recorded as a continuous variable and was derived from a logistic regression model with $\beta$ blocker use as a dichotomous dependent variable. We included all recorded demographic factors, co-morbid illnesses and treatments as potential covariates in the propensity score model. Variables were entered and maintained in the model if they had a $p$ value of $<0.20$ in the final model.

A multivariate logistic regression model including all patient characteristics as well as the propensity score was then optimised using backward selection and analysed to identify predictors of in-hospital mortality. Interaction terms between $\beta$ blocker use and each of the variables in the database were also included. Variables were omitted from the model if they were not significant and their removal altered the estimate of $\beta$ blocker effect by $<10 \%$. Additional models were developed to examine the relationship between the average number of daily doses of $\beta$ blockers and short-acting $\beta$ agonists and mortality. In an effort to address the possibility of confounding by indication, we also compared the point estimate for the effect of $\beta$ blockers with that of calcium channel blockers in mutually exclusive subgroups. Calculations were performed using SPSS V.15.0 statistical software and $p$ values $<0.05$ were considered significant.

\section{RESULTS}

A total of 825 patients admitted to University Hospital between 1 October 1999 and 30 September 2006 met the inclusion criteria. Table 1 reports the univariate comparison of characteristics according to $\beta$ blocker use.

Patients in both groups were predominantly Caucasian and there were a similar number of men and women. Most of the patients were admitted to a pulmonary subspecialty ward. Patients treated with $\beta$ blockers were older, had longer hospital stays and were more likely to have cardiovascular disease and greater total co-morbidity as measured by the Deyo-Charlson score. There was no difference in the frequency of respiratory failure or in-hospital mortality between the two groups.

Table 1 Characteristics of patients according to use of $\beta$ blockers

\begin{tabular}{lllc}
\hline Characteristic & $\begin{array}{l}\boldsymbol{\beta} \text { blockers } \\
\text { (N = 142) }\end{array}$ & $\begin{array}{l}\text { No } \boldsymbol{\beta} \text { blockers } \\
\text { (N = 683) }\end{array}$ & p Value \\
\hline Age (years)* & $68(11)$ & $65(13)$ & 0.01 \\
Female & $71(50)$ & $344(50)$ & 1 \\
Caucasian race & $82(58)$ & $429(63)$ & 0.30 \\
Number of prior admissions for AECOPD* & $1.4(1.1)$ & $1.6(1.6)$ & 0.10 \\
Mean length of stay (days)* & $7.8(12.9)$ & $5.3(7.3)$ & 0.001 \\
Deyo-Charlson index* & $2.4(1.3)$ & $1.9(1.4)$ & $<0.001$ \\
Co-morbidities & & & \\
$\quad$ Cardiovascular disease & $87(61)$ & $219(32)$ & $<0.001$ \\
$\quad$ Myocardial infarction & $22(15)$ & $48(7)$ & 0.002 \\
$\quad$ Congestive heart failure & $71(50)$ & $157(23)$ & $<0.001$ \\
$\quad 11(8)$ & $36(5)$ & 0.34 \\
$\quad$ Peripheral vascular disease & $12(8)$ & $22(3)$ & 0.01 \\
$\quad$ Malignancy & $5(4)$ & $35(5)$ & 0.55 \\
$\quad$ Diabetes mellitus & $36(25)$ & $126(18)$ & 0.08 \\
Chronic renal insufficiency & $10(7)$ & $52(8)$ & 0.86 \\
$\quad$ Chronic liver disease & $2(1)$ & $11(2)$ & 1 \\
Current smoking & $78(55)$ & $363(53)$ & 0.76 \\
Respiratory failure & $24(17)$ & $85(12)$ & 0.20 \\
Admitted to pulmonary specialist service & $82(58)$ & $404(59)$ & 0.82 \\
In-hospital mortality & $8(6)$ & $35(5)$ & 0.83 \\
\hline AECOPD, & &
\end{tabular}

AECOPD, acute exacerbation of chronic obstructive pulmonary disease.

Data are shown as * mean (SD) or $\mathrm{n}(\%)$. 
Table 2 Comparison of concomitant medication use in the two groups

\begin{tabular}{llll}
\hline Treatment & $\begin{array}{l}\boldsymbol{\beta} \text { blockers } \\
(\mathbf{N}=\mathbf{1 4 2})\end{array}$ & $\begin{array}{l}\text { No } \boldsymbol{\beta} \text { blockers } \\
(\mathbf{N}=\mathbf{6 8 3})\end{array}$ & p Value \\
\hline Systemic steroid & $130(92)$ & $634(93)$ & 0.73 \\
Inhaled steroid & $7(5)$ & $51(7)$ & 0.37 \\
Antibiotic & $128(90)$ & $585(86)$ & 0.20 \\
Short-acting $\beta$ agonist & $133(94)$ & $657(96)$ & 0.26 \\
Long-acting $\beta$ agonist & $8(6)$ & $37(5)$ & 0.92 \\
Short-acting anticholinergic & $132(93)$ & $647(95)$ & 0.53 \\
Long-acting anticholinergic & $2(1)$ & $6(1)$ & 0.63 \\
Methylxanthine & $11(8)$ & $111(16)$ & 0.01 \\
\hline
\end{tabular}

Values shown as $\mathrm{n}(\%)$.

Table 2 compares the concomitant medication use in the two groups. Patients who did not receive $\beta$ blockers were more likely to be treated with methlyxanthines (theophylline or aminophylline) but other medication use was similar between the two groups. Cardioselective $\beta$ blockers were administered to 121 patients (metoprolol and atenolol were most common) while 24 patients received non-cardioselective agents (carvedilol in 17).

Those who died were older than those who survived (71 vs 65 years, $p<0.001$ ), had longer hospital stays (17 vs 5 days, $\mathrm{p}<0.001)$ and were more often cared for on the pulmonary service $(79 \%$ vs $58 \%, p=0.006)$. Those who died also had more previous exacerbations $(2.7$ vs $1.5, \mathrm{p}<0.001)$ and were more likely to have cardiovascular disease (67\% vs $35 \%, \mathrm{p}<0.001)$, respiratory failure $(58 \%$ vs $11 \%, \mathrm{p}<0.001)$ and higher DeyoCharlson scores (2.5 vs 2.0, $\mathrm{p}=0.013$ ). Among those who died, care was withdrawn in 8 and an additional 26 had "do not resuscitate" orders prior to their deaths. While all 43 were admitted for acute exacerbations of COPD, the most frequent complications leading to death were nosocomial infection or progressive multiorgan failure $(n=12)$, myocardial infarction $(n=4)$ and renal failure $(n=4)$.

Predictors of in-hospital mortality are shown in table 3. The original model included all factors listed in tables 1 and 2 as well as the propensity score and interaction terms between the use of $\beta$ blockers and each of the other variables. No significant interactions were found. The $\mathrm{R}^{2}$ of the final adjusted model shown in table 3 was 0.38 . Age, increasing length of stay, respiratory failure and the number of prior acute exacerbations were associated with an increased risk of in-hospital death. The presence of congestive heart failure, cerebrovascular disease and liver disease also predicted increased mortality. The use of $\beta$ blockers (OR $=0.39 ; 95 \%$ CI 0.14 to 0.99 ) and short-acting $\beta$ agonists $(\mathrm{OR}=0.08 ; 95 \%$ CI 0.02 to 0.30$)$ were associated with reduced mortality. We also found a significant association between the number of daily doses of $\beta$ blocker and mortality $(\mathrm{OR}=0.31 ; 95 \%$ CI 0.12 to 0.80 ), although no dose-response relationship was observed with short-acting $\beta$ agonists $(\mathrm{OR}=1.02 ; 95 \%$ CI 0.70 to 1.47). The point estimates for the effect of $\beta$ blockers were similar when stratified for the presence or absence of cardiovascular disease (OR 0.37 vs 0.38 ), although neither reached statistical significance. The point estimate of the effect of $\beta$ blockers among those who did not receive calcium channel blockers ( $N=577)$ was comparable to that of the overall population $(\mathrm{OR}=0.34 ; 95 \% \mathrm{CI}$ 0.06 to 1.81$)$ although not statistically significant $(p=0.20)$. No association was seen between calcium channel blocker use and mortality in those who did not receive $\beta$ blockers $(N=683)$ $(\mathrm{OR}=0.76$; $95 \%$ CI 0.28 to $2.06, \mathrm{p}=0.60)$, indicating specificity of the $\beta$ blocker effect.

In the subset of patients with available spirometric data there was no significant difference between those who received $\beta$ blockers and those who did not in mean (SD) percentage predicted $\mathrm{FEV}_{1}$ (41 (16)\% vs $40(15) \%$; $=44$ vs $\left.240, \mathrm{p}=0.67\right)$ or $\mathrm{FEV}_{1} / \mathrm{FVC}$ ratio $(0.50(0.10)$ vs $0.53(0.08), \mathrm{p}=0.61)$.

\section{DISCUSSION}

This study, which examined a large population of inpatients admitted for acute exacerbations of COPD, found that $\beta$ blocker use was associated with reduced in-hospital mortality. When evaluated in a multivariate regression model including a propensity score, $\beta$ blocker use was a powerful predictor of outcome. The benefit of $\beta$ blockers was observed despite the fact that those who received the drugs were older, had longer hospital stays and had a greater prevalence of congestive heart failure and cerebrovascular disease, all factors that were independent predictors of in-hospital mortality. We found no association between calcium channel blocker use and hospital mortality, suggesting our results are not due to healthy user bias and that $\beta$ blockers may offer a class-specific benefit.

Table 3 Predictors of in-hospital mortality

\begin{tabular}{llclr}
\hline Parameter & $\begin{array}{l}\text { Unadjusted OR for death } \\
\text { (95\% Cl) }\end{array}$ & p Value & $\begin{array}{l}\text { Adjusted OR for death } \\
\text { (95\% CI) }\end{array}$ & p Value \\
\hline$\beta$ blocker use & $1.10(0.50$ to 2.44$)$ & 0.80 & $0.39(0.14$ to 0.99$)$ & 0.049 \\
Short-acting $\beta$ agonist use & $0.08(0.04$ to 0.17$)$ & $<0.001$ & $0.08(0.02$ to 0.30$)$ & $<0.001$ \\
Age (per year of life) & $1.05(1.02$ to 1.08$)$ & 0.001 & $1.05(1.02$ to 1.09$)$ & 0.004 \\
Number of prior AECOPD & $1.27(1.12$ to 1.44$)$ & $<0.001$ & $1.22(1.01$ to 1.47$)$ & 0.037 \\
Length of stay (per day) & $1.06(1.04$ to 1.09$)$ & $<0.001$ & $1.05(1.02$ to 1.08$)$ & $<0.001$ \\
Respiratory failure & $11.5(6.04$ to 22.0$)$ & $<0.001$ & $10.2(4.58$ to 22.6$)$ & $<0.001$ \\
Congestive heart failure & $3.58(1.92$ to 6.67$)$ & $<0.001$ & $4.54(1.53$ to 13.5$)$ & 0.006 \\
Cerebrovascular disease & $3.41(1.25$ to 9.32$)$ & 0.016 & $12.9(3.10$ to 53.3$)$ & $<0.001$ \\
Chronic liver disease & $3.42(0.73$ to 15.9$)$ & 0.12 & $12.1(2.06$ to 71.5$)$ & 0.006 \\
\hline
\end{tabular}

OR, odds ratio; $\mathrm{Cl}$, confidence interval; $\mathrm{AECOPD}$, acute exacerbation of chronic obstructive pulmonary disease. 
Beta-blockers are often withheld from patients with COPD because of the perception that their use is relatively contraindicated. ${ }^{19} 22$ This is despite the fact that cardioselective $\beta$ blockers have no demonstrable effect on lung function regardless of disease severity or bronchodilator reversibility, ${ }^{23}$ and that patients with COPD who have cardiac disease appear to derive the same benefits from these drugs as does the general population. ${ }^{19}$ Current American Heart Association guidelines for secondary prevention of coronary disease include $\beta$ blockers as a class IA intervention in patients with prior myocardial infarction, acute coronary syndrome or congestive heart failure, ${ }^{29}$ and the Centers for Medicaid and Medicare include $\beta$ blocker use following myocardial infarction as a hospital performance measure..$^{30}$ In our study only $31 \%$ of patients (93/298) with COPD and congestive heart failure or myocardial infarction received $\beta$ blockers. This is comparable to prior reports ${ }^{1922} 31$ that have also suggested that most patients with COPD who have cardiovascular disease are denied the protective effect offered by these agents.

No prospective studies have specifically examined the effect of $\beta$ blockers on mortality in a large cohort of patients with $\mathrm{COPD}$, but significant observational data support the safety and effectiveness of the drugs when used in this population. Gottlieb reviewed the medical records of all Medicare patients discharged after acute myocardial infarction over an 18-month period and evaluated survival with social security records. ${ }^{19}$ After adjustment for covariates, patients with COPD who received $\beta$ blockers had a $40 \%$ reduction in mortality which was similar to the reduction seen in the entire population. Au et $a^{24}$ examined the association between $\beta$ blocker use and all-cause mortality at 2 years in a veteran population with COPD and hypertension. Compared with calcium channel blockers, $\beta$ blockers were associated with a reduced risk of death (hazard ratio $(H R)=0.57)$. When compared with all other antihypertensive agents, the results were similar. Importantly, the benefit of $\beta$ blockers was limited to those with cardiac disease (absolute risk reduction $12.8 \%, \mathrm{p}<0.001)$ and not observed in those without (absolute risk reduction $4.0 \%, p=0.37$ ), although the study may have been underpowered to detect this small difference. It is also notable that there was actually a trend towards reduced COPD-related hospitalisations among those taking $\beta$ blockers ( $H R=0.46$; CI 0.21 to 1.04). Despite the limitations of their retrospective design, these studies suggest a mortality benefit with the use of $\beta$ blockers in patients with COPD who have had a recent myocardial infarction or have other cardiac disease.

Other data indicate that $\beta$ blockers may improve outcomes in patients with COPD who do not have known cardiac disease. Mancini et $a l^{32}$ showed that patients with COPD who suffered a myocardial infarction and were hospitalised for COPD or died were less likely to be taking $\beta$ blockers regardless of their risk for cardiovascular events. Furthermore, Au et al ${ }^{12}$ showed that $\beta$ blockers reduced the risk of cardiovascular events even after adjusting for the presence of cardiovascular disease and other risk factors such as smoking, hypertension and diabetes mellitus.

Previous studies have implicated cardiovascular disease as an independent risk factor for hospitalisation for COPDD ${ }^{33}$ inhospital mortality ${ }^{15}{ }^{16}$ and mortality after discharge. ${ }^{77}$ In an analysis of 1829 veterans participating in a randomised trial comparing tiotropium with placebo, Niewoehner et a ${ }^{133}$ found that any cardiovascular disease was an independent risk factor for hospitalisation for acute exacerbation $(\mathrm{OR}=2.10$; CI 1.28 to $3.46, \mathrm{p}=0.004)$. Ai-Ping et $a l^{16}$ also reported that non-survivors of an intensive care unit admission for acute exacerbations were more likely than survivors to have cardiac disease ( $85 \%$ vs $37 \%$, $p=0.002$ ). Similarly, in a multivariate analysis of factors associated with in-hospital death during acute exacerbations, Fuso et $a l^{15}$ found that variables reflecting cardiovascular dysfunction were among the key determinants of risk.

In addition to the apparent protective effect of $\beta$ blockers, we found that treatment with short-acting $\beta$ agonists was associated with reduced mortality. This finding is unexpected based on prior reports suggesting that these agents may increase the risk of cardiovascular morbidity when used chronically in the outpatient setting. ${ }^{10-12}$ It is possible that this result reflects the avoidance of short-acting $\beta$ agonists among patients with the highest risk of death such as those with tachyarrhythmias or other unstable cardiac disease, rather than a true beneficial effect among recipients. Indeed, although all 43 patients who died were treated with short-acting anticholinergic agents, 12 were not prescribed short-acting $\beta$ agonists which suggests that practitioners may have judged them to be unsafe. Of note, the benefit of short-acting $\beta$ agonists was lost when the number of daily doses was examined as the independent variable. When combined with the analysis suggesting a dose-response benefit to $\beta$ blocker use, these results suggest that $\beta$ stimulation, whether endogenous or exogenous, may be deleterious for patients with acute exacerbations of COPD. Despite this hypothesis, it remains possible that $\beta$ agonists are beneficial for inpatients with severe exacerbations as they are known to improve lung function and symptoms rapidly in that setting. ${ }^{34}$ We found no evidence that $\beta$ blockers reduce the beneficial effects of short-acting $\beta$ agonists when the two are used concomitantly during acute exacerbations. It has been suggested that $\beta$ blockers may in fact improve bronchodilator responsiveness by leading to upregulation of $\beta$ receptors within the lung, ${ }^{35}$ although this requires further study.

Although the use of $\beta$ blockers was an independent predictor of survival in multivariate analysis, univariate analysis did not demonstrate a statistically significant mortality advantage to their use. This underscores the importance of adjusting for the covariates in the model, particularly given the greater comorbidity found in $\beta$ blocker users.

As a retrospective study, this report has important limitations. Although the multivariate analysis can correct for known covariates, limitations in the database may confound the results. For example, it is possible that patients who received $\beta$ blockers had less severe baseline COPD or less serious exacerbations than those who did not. However, the lack of difference between the two groups in the number of prior admissions for acute exacerbations or frequency of respiratory failure suggests this was not the case. The available pulmonary function data also suggest that the severity of airflow obstruction was similar between the two groups. Although other investigators have used pharmacy billing records to measure inpatient medication use, ${ }^{36}{ }^{37}$ Ward et a ${ }^{18}$ have shown that point of care clinical information systems may capture inpatient medication use more accurately than pharmacy records as they are more closely connected to nursing and respiratory care. This may be particularly true for bronchodilators as they are often administered by staff on an "as needed" basis. The use of inpatient billing records also prevented the evaluation of outpatient medication use, dosage or adherence, and it is not known whether $\beta$ blockers were started in the hospital or continued based on outpatient prescriptions. There may be differences in the effects of chronic $\beta$ blocker use and the new administration of these drugs during acute exacerbations, 
and this warrants further investigation. The acute withdrawal of $\beta$ blockers has been associated with an increased risk of myocardial infarction, ${ }^{39}$ and it is possible that our results could be partly explained by adverse effects of this practice among patients whose $\beta$ blockers were discontinued on admission. Lastly, larger studies are needed to better define whether the mortality benefit of $\beta$ blockers in this setting is observed with cardioselective agents, non-cardioselective agents, or both.

In summary, the results of this study suggest that the use of $\beta$ blockers in patients admitted with acute exacerbations of COPD is not deleterious and may be associated with a beneficial effect on mortality. These results have direct implications for the use of $\beta$ blockers in patients hospitalised for acute exacerbations of COPD and suggest that they can be safely continued in this setting. The strength of these and other observational data supporting the safety and potential efficacy of $\beta$ blockers in patients with COPD now favours the pursuit of randomised trials in the outpatient setting. If our results are confirmed, prospective trials among inpatients with acute exacerbations should also be considered.

Acknowledgements: The authors thank Darlene Green for her assistance with data abstraction.

Funding: SMR is supported by NIH grant K23 DK075788-01.

Competing interests: None.

\section{REFERENCES}

1. Mannino DM.COPD: epidemiology, prevalence, morbidity and mortality, and disease heterogeneity. Chest 2002;121(suppl):121-6S

2. Vilkman S, Keistinen T, Tuuponen T, et al. Survival and cause of death among elderly chronic obstructive pulmonary disease patients after first admission to hospital. Respiration 1997;64:281-4.

3. Kuller LH, Ockene JK, Townsend M, et al. The epidemiology of pulmonary function and COPD mortality in the multiple risk factor intervention trial. Am Rev Respir Dis 1989;140:S76-81.

4. Tockman MS, Comstock GW. Respiratory risk factors and mortality: Iongitudinal studies in Washington County, Maryland. Am Rev Respir Dis 1989;140:S56-63.

5. Almagro P, Calbo E, Ochoa de Echagiien A, et al. Mortality after hospitalization for COPD. Chest 2002;121:1441-8.

6. Anthonisen NR, Skeans MA, Wise RA, et al. The effects of a smoking cessation intervention on 14.5-year mortality: a randomized clinical trial. Ann Intern Med 2005; 142:233-9.

7. Antonelli Incalzi RA, Fuso L, De Rosa M, et al. Co-morbidity contributes to predict mortality of patients with chronic obstructive pulmonary disease. Eur Respir $J$ 1997; 10:2794-800.

8. Thurnheer R, Muntwyler J, Stammberger U, et al. Coronary disease in patients undergoing lung volume reduction surgery for emphysema. Chest 1997:112:122-8

9. Man SF, Sin DD. Effects of corticosteroids on systemic inflammation in chronic obstructive pulmonary disease. Proc Am Thorac Soc 2005;2:78-82.

10. Suissa S, Hemmelgarn B, Blais $L$, et al. Bronchodilators and acute cardiac death. Am J Respir Crit Care Med 1996;154:1598-602.

11. Au DH, LeMaitre RN, Curtis JR, et al. The risk of myocardial infarction associated with inhaled beta-adrenoceptor agonists. Am J Respir Crit Care Med 2000;161:82730.

12. Au DH, Curtis JR, Every NR, et al. Association between inhaled beta-agonists and the risk of unstable angina and myocardial infarction. Chest 2002;121:846-51.

13. Patil SP, Krishnan JA, Lechtzin N, et al. In-hospital mortality following acute exacerbations of chronic obstructive pulmonary disease. Arch Intern Med 2003;163:1180-6.

14. Donaldson GC, Wedzicha JA. COPD exacerbations. 1: Epidemiology. Thorax 2006;61:164-8.
15. Fuso L, Incalzi RA, Pistelli R, et al. Predicting mortality of patients hospitalized for acutely exacerbated chronic obstructive pulmonary disease. Am J Med 1995;98:272-7.

16. Ai-Ping C, Lee KH, Lim TK. In-hospital and 5-year mortality of patients treated in the ICU for acute exacerbation of COPD: a retrospective study. Chest 2005;128:518-24.

17. Connors AF Jr, Dawson NV, Thomas C, et al. Outcomes following acute exacerbation of severe chronic obstructive lung disease. The SUPPORT investigators (Study to Understand Prognoses and Preferences for Outcomes and Risks of Treatments). Am J Respir Crit Care Med 1996;154:959-67.

18. Seneff MG, Wagner DP, Wagner RP, et al. Hospital and 1-year survival of patients admitted to intensive care units with acute exacerbation of chronic obstructive pulmonary disease. JAMA 1995;274:1852-7.

19. Gottlieb SS, McCarter RJ, Vogel RA. Effect of beta-blockade on mortality among high-risk and low-risk patients after myocardial infarction. $N$ Engl J Med 1998;339:489-97.

20. Cleophas TJ, Swinderman AH. Beta-blockers and heart failure: meta-analysis of mortality trials. Int J Clin Pharmacol Ther 2001;39:383-8.

21. Mangano DT, Layug EL, Wallace A, et al. Effect of Atenolol on mortality and cardiovascular morbidity after noncardiac surgery. N Engl J Med 1996;335:1713-20.

22. Egred M, Shaw S, Mohammed B, et al. Under-use of beta-blockers in patients with ischaemic heart disease and concomitant chronic obstructive pulmonary disease. Q J Med 2005;98:493-7.

23. Salpeter S, Omiston T, Salpeter E. Cardioselective beta-blockers for chronic obstructive pulmonary disease. Cochrane Database Syst Rev 2005;(4):CD003566.

24. Au DH, Bryson CL, Fan VS, et al. Beta-blockers as single-agent therapy for hypertension and the risk of mortality among patients with chronic obstructive pulmonary disease. Am J Med 2004;117:925-31.

25. van der Woude HJ, Zaagsma J, Postma DS, et al. Detrimental effects of betablockers in COPD: a concern for nonselective beta-blockers. Chest 2005;127:818-24.

26. Kotlyar $\mathbf{E}$, Keogh AM, Macdonald PS, et al. Tolerability of carvedilol in patients with heart failure and concomitant chronic obstructive pulmonary disease or asthma. $J$ Heart Lung Transplant 2002;21:1290-5.

27. Deyo RA, Cherkin DC, Ciol MA. Adapting a clinical comorbidity index for use with ICD-9-CM administrative databases. J Clin Epidemiol 1992;45:613-9.

28. Stone RA, Obrosky DS, Singer DE, et al. Propensity score adjustment for pretreatment differences between hospitalized and ambulatory patients with community-acquired pneumonia. Pneumonia Patient Outcomes Research Team (PORT) Investigators. Med Care 1995;33(4 Suppl):AS56-66.

29. Smith SC Jr, Allen J, Blair SN, et al. AHA/ACC guidelines for secondary prevention for patients with coronary and other atherosclerotic vascular disease: 2006 update: endorsed by the National Heart, Lung, and Blood Institute. Circulation 2006;113:2363-72.

30. Anon. http://www.cms.hhs.gov/HospitalQualitylnits/downloads/ HospitalOverviewOfSpecs200512.pdf (accessed 22 March 2007).

31. Sinha S, Goldstein M, Penrod J, et al. Brief report: beta-blocker use among veterans with systolic heart failure. J Gen Intern Med 2006;21:1306-9.

32. Mancini GB, Etminan M, Zhang B, et al. Reduction of morbidity and mortality by statins, angiotensin-converting enzyme inhibitors, and angiotensin receptor blockers in patients with chronic obstructive pulmonary disease. J Am Coll Cardiol 2006;47:2554-60.

33. Niewoehner DE, Lokhnygina $Y$, Rice $K$, et al. Risk indexes for exacerbations and hospitalizations due to COPD. Chest 2007;131:20-8.

34. Rodriguez-Roisin R. COPD exacerbations. 5: Management. Thorax 2006;61:53544.

35. Ormiston TM, Salpeter SR. Beta-blocker use in patients with congestive heart failure and concomitant obstructive airway disease: moving from myth to evidence-based practice. Heart Fail Monit 2003;4:45-54.

36. Marill KA, deSouza IS, Nishijima DK, et al. Amiodarone is poorly effective for the acute termination of ventricular tachycardia. Ann Emerg Med 2006;47:217-24.

37. Ury WA, Rahn M, Tolentino $\mathrm{V}$, et al. Can a pain management and palliative care curriculum improve the opioid prescribing practices of medical residents? J Gen Intern Med 2002;17:625-31.

38. Ward NS, Snyder JE, Ross S, et al. Comparison of a commercially available clinical information system with other methods of measuring critical care outcomes data. J Crit Care 2004;19:10-5.

39. Psaty BM, Koepsell TD, Wagner EH, et al. The relative risk of incident coronary heart disease associated with recently stopping the use of beta-blockers. JAMA 1990;263:1653-7. 